\title{
FGF21 as a hepatokine, adipokine, and myokine in metabolism and diseases
}

\section{Nobuyuki Itoh*}

Kyoto University Graduate School of Pharmaceutical Sciences, Kyoto, Japan

Edited by:

Gaetano Santulli, Columbia

University, USA

\section{Reviewed by:}

Encarnación Capilla, University of

Barcelona, Spain

Marily Theodoropoulou, Max Planck

Institute of Psychiatry, Germany

Dubravka Jurišic Eržen, Clinical

Hospital Centre Rijeka, Croatia

Sahar Mohamed Kamal, Ain Shams

University, Egypt

Alexandra Proshchina, Russian

Academy of Medical Sciences, Russia

${ }^{*}$ Correspondence:

Nobuyuki Itoh, Kyoto University Graduate School of Pharmaceutical

Sciences, Yoshida-shimoadachi,

Sakyo, Kyoto 606-8501, Japan

e-mail: itohnobu@pharm.kyoto-u.

ac.jp
Fibroblast growth factor (FGF) family members are mostly secreted as signaling proteins with diverse functions in development and metabolism. FGF21 is a unique FGF with metabolic, but not proliferative activities. FGF21 is mostly induced by different kinds of stress and acts though FGF receptor $1 \mathrm{c}$ with $\beta$-Klotho as a cofactor in an endocrine or, in parts, autocrine/paracrine manner. Hepatic FGF21 directly acts on white adipocytes to inhibit lipolysis and acts through the brain to increase systemic glucocorticoid levels and suppress physical activity in response to starvation. It also protects against dioxin toxicity. Adipocytic FGF21 induces the browning of white adipose tissue (WAT) and activates brown adipocytes in response to cold exposure. It also acts as an upstream effector of adiponectin in white adipocytes. Myocytic FGF21 protects against diet-induced obesity and insulin resistance, induces the browning of WAT, and protects against cardiac hypertrophy. In addition, Fgf21 polymorphisms are possibly related with metabolic diseases and FGF21 are biomarker of metabolic diseases. These findings indicate that FGF21 plays roles as a hepatokine, adipokine, and myokine in metabolism, injury protection, and diseases.

Keywords: adipokine, biomarker, disease, FGF21, hepatokine, metabolism, myokine, stress

\section{INTRODUCTION}

Fibroblast growth factors (FGFs) are signaling proteins of $\sim 150$ 300 amino acids with diverse biological functions mainly in development and metabolism. The human/mouse FGF family comprises FGF1-FGF23. However, as mouse FGF15 and human FGF19 are orthologs, they are usually referred to as FGF15/19 (1). FGFs can be classified into paracrine, intracrine, and endocrine FGFs based on their mechanisms of action. Paracrine FGFs mainly function as secreted local signaling molecules in development. Endocrine FGFs mainly function as hormone-like or, in parts, local signaling molecules in metabolism. In contrast, intracrine FGFs mainly function as intracellular molecules in neuronal processes (1).

Endocrine FGFs comprise FGF15/19, FGF21, and FGF23 (1). Although most of paracrine and endocrine FGFs have proliferative activities, FGF21 is a unique FGF with metabolic, but not proliferative activities. FGF21 exerts diverse pharmacological effects on metabolism. Review articles that focus on its pharmacological effects and therapeutic uses for metabolic diseases have already been published $(2,3)$. This succinct review focuses on the physiological and pathophysiological roles of FGF21 as a metabolic regulator.

\section{MECHANISIM OF ACTION OF FGF21}

Paracrine and endocrine FGF signaling is mostly mediated by the activation of FGF receptors (FGFRs) including FGFRs 1b, $1 c, 2 b, 2 c, 3 b, 3 c$, and 4 with different ligand-binding specificities. FGFs activate four key intracellular signaling pathways: RAS/RAF/mitogen-activation protein kinase (MAPK), phosphatidylinositol 3-kinase (PI3K)/serine-threonine protein kinase
AKT, signal transducer and activator of transcription (STAT), and phosphoinositide phospholipase C (PLC) $\gamma$ (1). Endocrine FGFs activate FGFRs with $\alpha$-Klotho or $\beta$-Klotho as a cofactor, which are specifically expressed in their targeted tissues (1). FGF21 activates FGFR1c with $\beta$-Klotho $(1,4)$.

\section{FGF21 AS A HEPATOKINE}

Fgf21 is abundantly expressed in the liver. Hepatic Fgf21 expression was possibly induced during fasting through the activation of peroxisome proliferator-activated receptor (PPAR) $\alpha$ by nonesterified fatty acids that are released from adipocytes and taken up by hepatocytes (5). Lipolysis was increased in the white adipocytes of fasted Fgf21 knockout mice, indicating that FGF21 inhibit lipolysis during fasting (Table 1) (6). A low-carbohydrate, high-fat ketogenic diet (KD) significantly induced hepatic Fgf21 expression. The impaired insulin sensitivity in WAT caused by KD feeding was improved in the knockout mice, indicating that FGF21 is a negative regulator of adipocyte insulin sensitivity in adaptation to a low-carbohydrate malnutritional state (7). FGF21 also increased systemic glucocorticoid levels and suppressed physical activity in adaptation to the starvation response. Mice lacking $\beta$-Klotho in the suprachiasmatic nucleus and dorsal vagal complex of the brain were refractory to these effects. Thus, hepatic FGF21 also exerts diverse actions through $\beta$-Klotho in the brain (Table 1) (8).

In addition to fasting and $\mathrm{KD}$, hepatic $F g f 21$ expression was significantly induced by different kinds of stress such as hepatic injury, chemical insult, and diseases. Hepatic FGF21 is a stressinduced metabolic regulator (19). The toxicity of dioxins is also well documented. Dioxins increased hepatic Fgf21 expression and 
Table 1 | Functions of FGF21 as a hepatokine, adipokine, and myokine.

\begin{tabular}{llll}
\hline FGF21 & Targeted tissues & Functions & Action manners \\
\hline Hepatokine & WAT & Lipid metabolism & Endocrine \\
& Brain & Glucocorticoid metabolism, physical & Endocrine \\
& Not identified & Protection against dioxin toxicity & Endocrine \\
Adipokine & WAT & Thermogenesis & Autocrine/paracrine \\
& BAT & Adiponectin induction & Autocrine/paracrine \\
WyT & Thermogenesis & Autocrine/paracrine \\
Skeletal muscle & Energy metabolism, thermogenesis & Endocrine \\
(11), 12) & Autocrine/paracrine
\end{tabular}

WAT, white adipose tissue; BAT, brown adipose tissue.

serum FGF21 levels. Its toxicity was enhanced in Fgf21 knockout mice, indicating that FGF21 protect against this toxicity (Table 1) (9).

\section{FGF21 AS AN ADIPOKINE}

Uncoupling protein 1 (UCP1) releases chemical energy as heat in brown adipocytes. Beige adipocytes, brown adipocyte-like cells, are UCP1-positive adipocytes in white adipose tissue (WAT). Beige adipocytes markedly accumulated in subcutaneous WAT following cold exposure (20). Cold exposure induced Fgf21 expression in WAT expressing Fgfrlc and $\beta$-Klotho. The accumulation of beige adipocytes was impaired in Fgf21 knockout mice, indicating that FGF21 induces the accumulation of beige adipocytes in WAT in an autocrine/paracrine manner (Table 1) (10). Cold exposure also induced $F g f 21$ expression in brown adipocytes expressing Fgfr1c and $\beta$-Klotho. FGF21 also activates brown adipocytes in an autocrine/paracrine manner (Table 1) $(13,14)$. In humans, cold exposure increased circulating FGF21 levels, which activated brown adipocytes and enhanced lipolysis and thermogenesis response through browning of $\operatorname{WAT}(21,22)$. Thus, FGF21 activates the thermogenic machinery against hypothermia.

The systemic administration of FGF21 had no effect on metabolism in lipodystrophic mice with a WAT deficiency. However, the administration of FGF21 was effective in these mice following the transplantation of WAT (23). Thus, WAT is the predominant site that confers the metabolic activities of FGF21. Adiponectin, an adipokine produced in WAT, controls systemic glucose and lipid homeostasis in the liver and skeletal muscle in an endocrine manner. FGF21 has many functional similarities to adiponectin. Furthermore, FGF21 produced in WAT enhanced the expression of adiponectin in WAT as well as serum adiponectin levels. Several therapeutic benefits of FGF21 were impaired in adiponectin knockout mice. The effects of FGF21 on the attenuation of obesity-induced impairments in insulin signaling in the liver and skeletal muscle were also impaired in adiponectin knockout mice. Thus, adiponectin acts as a downstream effector of FGF21 in WAT and mediates the effects of FGF21 on energy metabolism and insulin sensitivity in the liver and skeletal muscle (Table 1) (11, 12). Insulin resistance develops in insulinresponsive tissues due to the aberrant accumulation of intracellular lipids including the sphingolipid ceramide. FGF21 diminished the accumulation of ceramides in obese animals. Changes in energy expenditure and the ceramide-lowering effects induced by FGF21 were impaired in adiponectin knockout mice. Thus, the FGF21-adiponectin-ceramide axis controls energy expenditure and insulin action (12).

\section{FGF21 AS A MYOKINE}

The PI3K/Akt1 pathway has been implicated in insulin signaling and cellular hypertrophy. Skeletal muscle fiber hypertrophy was observed in skeletal muscle-specific Akt1 transgenic mice. The expression of Fgf21 in the muscle and serum FGF21 levels was increased in the Akt1 transgenic mice (24). Thus, skeletal muscle is also a source of FGF21, the expression of which is regulated by a PI3K/Akt1 signaling pathway-dependent mechanism.

FGF21 is also known to be a myokine that is induced by different kinds of stress (19). Cytoplasmic constituents are delivered to lysosomes for the degradation of aggregated proteins and recycling of organelles or nutrients by autophagy. The amino acids produced by autophagy are used for energy production or other purposes during nutrient deficiencies. Mitochondrial dysfunction in autophagy-related 7 (Atg7) knockout mice increased Fgf21 expression by activating transcription factor 4 (Atf4), a master regulator of the integrated stress response (15). An autophagy deficiency and subsequent mitochondrial dysfunction increased the production of FGF21 as a myokine to promote protection against diet-induced obesity and insulin resistance (Table 1). The induction of Fgf21, resistance to diet-induced obesity, and amelioration of insulin resistance were also observed in the livers of mice with autophagy deficiencies (15).

Fgf21 was induced in the skeletal muscle of Ucp1 skeletal muscle-specific transgenic mice and resulted in significantly 
elevated serum FGF21 levels. The integrated stress response was activated in the skeletal muscle of Ucp1 transgenic mice without myopathy or a muscle autophagy deficiency. The browning of WAT with lipolysis and respiratory capacity was also increased in Ucp 1 transgenic mice (16). Improved substrate metabolism and increased longevity were also observed in Ucp 1 transgenic mice. Targeting mitochondrial function in cultured myoblasts by treatments with respiratory chain inhibitors resulted in the activation of an integrated stress response associated with the increased expression of $F g f 21$. In addition, white adipocytes cultured with the serum of $U c p 1$ transgenic mice resulted in an increase in the expression of $U c p 1$. These findings indicate that the production of FGF21 is coupled to mitochondrial dysfunction and the activation of an integrated stress response in skeletal muscle. Thus, FGF21 as a myokine exhibits effects that lead to the increased browning of WAT in an endocrine manner (Table 1) (16). A subgroup of human immunodeficiency virus (HIV) patients is associated with insulin resistance. $F g f 21$ expression in skeletal muscle, but not serum FGF21 levels was increased in HIV patients with lipodystrophy and this was correlated to insulin resistance, indicating that FGF21 is a myokine that may be correlated with insulin resistance in an autocrine/paracrine manner (Table 1) (17).

Exaggerated cardiac hypertrophy and dysfunction were observed in Fgf21 knockout mice in response to the infusion of isoproterenol. Cardiomyocytes produced and secreted FGF21, indicating that FGF21 is a myokine that may protect against cardiac hypertrophy in an autocrine/paracrine manner (Table 1) (18).

\section{FGF21 SINGLE NUCLEOTIDE POLYMORPHISMS AS RISK FACTORS FOR DISEASES}

The intake of dietary macronutrients is associated with an increased risk of obesity and diabetes. A single nucleotide polymorphism (SNP) in the Fgf21 exon was correlated with the percentage of total caloric intake from protein and carbohydrate, suggesting that FGF21 is a potentially susceptible gene for obesity and diabetes (25). SNPs in the Fgf21 $3^{\prime}$ non-coding region were also associated with metabolic syndrome, obesity, and diabetes (26) (Table 2). These findings indicate that Fgf21 SNPs are possibly related with metabolic diseases.

\section{SERUM FGF21 LEVELS AS BIOMARKERS OF DISEASES}

Coronary heart disease (CHD) with a narrowing of the small blood vessels in the heart could lead to heart attacks. Serum FGF21 levels were significantly higher in CHD patients, and were even higher in CHD patients with diabetes, and hypertension. However, high serum FGF21 levels were associated with adverse lipid profiles in CHD patients, indicating that this paradoxical increase in CHD patients may reflect a compensatory response or resistance to FGF21 (27). Carcinoid atherosclerosis typically results in carotid stenosis. Serum FGF21 levels were positively correlated with carotid intima-media thickness (IMT) in women (28). Serum FGF21 levels were also significantly higher in patients with both obesity and type 2 diabetes (29), in mitochondria disease patients with dysfunctional mitochondria (30), in Cushing's syndrome patients with prolonged and inappropriately high levels of glucocorticoids (31), and in preeclampsia patients with serious cardiovascular complications in pregnancy (32). In contrast,
Table 2 | FGF21 as a risk factor for and biomarker of diseases.

\begin{tabular}{|c|c|c|}
\hline FGF21 & Diseases & References \\
\hline \multicolumn{3}{|l|}{ RISK FACTOR } \\
\hline SNP in the exon & Dietary macronutrient intake & $(25)$ \\
\hline $\begin{array}{l}\text { SNPS in the } 3^{\prime} \\
\text { non-coding region }\end{array}$ & $\begin{array}{l}\text { Metabolic syndrome, obesity, } \\
\text { Type } 2 \text { diabetes }\end{array}$ & $(26)$ \\
\hline
\end{tabular}

\begin{tabular}{lll} 
BIOMARKER & \\
Increased serum levels & Coronary heart disease \\
Increased serum levels & Carcinoid atherosclerosis \\
Increased serum levels & Obesity, type 2 diabetes \\
Increased serum levels & Mitochondrial disease \\
Increased serum levels & Cushing's syndrome \\
Increased serum levels & Preeclampsia \\
Decreased serum levels & Anorexia nervosa \\
\hline
\end{tabular}

SNP, single nucleotide polymorphism.

serum FGF21 levels were decreased in anorexia nervosa patients with prominent reductions in body weight due to eating disorder (33) (Table 2). These findings indicate that serum FGF21 levels may be used as biomarkers of these diseases.

\section{CONCLUSION}

FGF21 acts in an FGFR-dependent manner with $\beta$-Klotho as a cofactor. FGF21 with metabolic, but not proliferative activities has unique functions. FGF21, which is typically induced by different kinds of stress, plays various roles in energy metabolism and injury protection as a hepatokine, adipokine, and myokine in an endocrine or autocrine/paracrine manner. Fgf21 single polymorphisms are possibly related with metabolic diseases and FGF21 and biomarker of metabolic diseases. These findings provide new insights into the physiological and pathophysiological roles of FGF21.

\section{REFERENCES}

1. Itoh N, Ornitz DM. Fibroblast growth factors: from molecular evolution to roles in development, metabolism and disease. J Biochem (2011) 149:121-30. doi:10.1093/jb/mvq121

2. Zhang J, Li Y. Fibroblast growth factor 21, the endocrine FGF pathway and novel treatments for metabolic syndrome. Drug Discov Today (2014) 19(5):579-89. doi:10.1016/j.drudis.2013.10.021

3. Kharitonenkov A, Adams AC. Inventing new medicines: the FGF21 story. Mol Metab (2014) 3:221-9. doi:10.1016/j.molmet.2013.12.003

4. Adams AC, Yang C, Coskun T, Cheng CC, Gimeno RE, Luo Y, et al. The breadth of FGF21's metabolic actions are governed by FGFR1 in adipose tissue. Mol Metab (2012) 2:31-7. doi:10.1016/j.molmet.2012.08.007

5. Murata Y, Konishi M, Itoh N. FGF21 as an endocrine regulator in lipid metabolism: from molecular evolution to physiology and pathophysiology. J Nutr Metab (2011) 2011:981315. doi:10.1155/2011/981315

6. Hotta Y, Nakamura H, Konishi M, Murata Y, Takagi H, Matsumura S, et al. Fibroblast growth factor 21 regulates lipolysis in white adipose tissue but is not required for ketogenesis and triglyceride clearance in liver. Endocrinology (2009) 150:4625-33. doi:10.1210/en.2009-0119 
7. Murata Y, Nishio K, Mochiyama T, Konishi M, Shimada M, Ohta H, et al. Fgf21 impairs adipocyte insulin sensitivity in mice fed a low-carbohydrate, high-fat ketogenic diet. PLoS One (2013) 8:e69330. doi:10.1371/journal.pone.0069330

8. Bookout AL, de Groot MH, Owen BM, Lee S, Gautron L, Lawrence HL, et al. FGF21 regulates metabolism and circadian behavior by acting on the nervous system. Nat Med (2013) 19:1147-52. doi:10.1038/nm.3249

9. Cheng X, Vispute SG, Liu J, Cheng C, Kharitonenkov A, Klaassen CD. Fibroblast growth factor (Fgf) 21 is a novel target gene of the aryl hydrocarbon receptor (AhR). Toxicol Appl Pharmacol (2014) 278:65-71. doi:10.1016/j. taap.2014.04.013

10. Fisher FM, Kleiner S, Douris N, Fox EC, Mepani RJ, Verdeguer F, et al. FGF21 regulates PGC- $1 \alpha$ and browning of white adipose tissues in adaptive thermogenesis. Genes Dev (2012) 26:271-81. doi:10.1101/gad.177857.111

11. Lin Z, Tian H, Lam KS, Lin S, Hoo RC, Konishi M, et al. Adiponectin mediates the metabolic effects of FGF21 on glucose homeostasis and insulin sensitivity in mice. Cell Metab (2013) 17:779-89. doi:10.1016/j.cmet.2013.04.005

12. Holland WL, Adams AC, Brozinick JT, Bui HH, Miyauchi Y, Kusminski CM, et al. An FGF21-adiponectin-ceramide axis controls energy expenditure and insulin action in mice. Cell Metab (2013) 17:790-7. doi:10.1016/j.cmet.2013.03.019

13. Fisher FM, Estall JL, Adams AC, Antonellis PJ, Bina HA, Flier JS, et al. Integrated regulation of hepatic metabolism by fibroblast growth factor 21 (FGF21) in vivo. Endocrinology (2011) 152:2996-3004. doi:10.1210/en.2011-0281

14. Hondares E, Iglesias R, Giralt A, Gonzalez FJ, Giralt M, Mampel T, et al. Thermogenic activation induces FGF21 expression and release in brown adipose tissue. J Biol Chem (2011) 286:12983-90. doi:10.1074/jbc.M110.215889

15. Kim KH, Jeong YT, Oh H, Kim SH, Cho JM, Kim YN, et al. Autophagy deficiency leads to protection from obesity and insulin resistance by inducing Fgf21 as a mitokine. Nat Med (2013) 19:83-92. doi:10.1038/nm.3014

16. Keipert S, Ost M, Johann K, Imber F, Jastroch M, Van Schothorst EM. Skeletal muscle mitochondrial uncoupling drives endocrine cross-talk through induction of FGF21 as a myokine. Am J Physiol Endocrinol Metab (2014) 306:E469-82. doi:10.1152/ajpendo.00330.2013

17. Lindegaard B, Hvid T, Grøndahl T, Frosig C, Gerstoft J, Hojman P, et al. Expression of fibroblast growth factor-21 in muscle is associated with lipodystrophy, insulin resistance and lipid disturbances in patients with HIV. PLoS One (2013) 8:e55632. doi:10.1371/journal.pone.0055632

18. Planavila A, Redondo I, Hondares E, Vinciguerra M, Munts C, Iglesias R, et al. Fibroblast growth factor 21 protects against cardiac hypertrophy in mice. Nat Commun (2013) 4:2019. doi:10.1038/ncomms3019

19. Luo Y, McKeehan WL. Stressed liver and muscle call on adipocytes with FGF21. Front Endocrinol (Lausanne) (2013) 4:194. doi:10.3389/fendo.2013.00194

20. Barbatelli G, Murano I, Madsen L, Hao Q, Jimenez M, Kristiansen K, et al. The emergence of cold-induced brown adipocytes in mouse white fat depots is determined predominantly by white to brown adipocyte transdifferentiation. Am J Physiol Endocrinol Metab (2010) 298:E1244-53. doi:10.1152/ajpendo.00600. 2009

21. Lee P, Linderman JD, Smith S, Brychta RJ, Wang J, Idelson C, et al. Irisin and FGF21 are cold-induced endocrine activators of brown fat function in humans. Cell Metab (2014) 19:302-9. doi:10.1016/j.cmet.2013.12.017

22. Lee P, Brychta RJ, Linderman J, Smith S, Chen KY, Celi FS. Mild cold exposure modulates fibroblast growth factor 21 (FGF21) diurnal rhythm in humans: relationship between FGF21 levels, lipolysis, and cold-induced thermogenesis. J Clin Endocrinol Metab (2013) 98:E98-102. doi:10.1210/jc.2012-3107
23. Véniant MM, Hale C, Helmering J, Chen MM, Stanislaus S, Busby J, et al. FGF21 promotes metabolic homeostasis via white adipose and leptin in mice. PLoS One (2012) 7:e40164. doi:10.1371/journal.pone.0040164

24. Izumiya Y, Bina HA, Ouchi N, Akasaki Y, Kharitonenkov A, Walsh K. FGF21 is an Akt-regulated myokine. FEBS Lett (2008) 582:3805-10. doi:10.1016/j.febslet. 2008.10.021

25. Chu AY, Workalemahu T, Paynter NP, Rose LM, Giulianini F, Tanaka T, et al. Novel locus including FGF21 is associated with dietary macronutrient intake. Hum Mol Genet (2013) 22:1895-902. doi:10.1093/hmg/ddt032

26. Zhang M, Zeng L, Wang YJ, An ZM, Ying BW. Associations of fibroblast growth factor 21 gene 3' untranslated region single-nucleotide polymorphisms with metabolic syndrome, obesity, and diabetes in a Han Chinese population. DNA Cell Biol (2012) 31:547-52. doi:10.1089/dna.2011.1302

27. Lin Z, Wu Z, Yin X, Liu Y, Yan X, Lin S, et al. Serum levels of FGF-21 are increased in coronary heart disease patients and are independently associated with adverse lipid profile. PLoS One (2010) 5:e15534. doi:10.1371/journal.pone.0015534

28. Chow WS, Xu A, Woo YC, Tso AW, Cheung SC, Fong CH, et al. Serum fibroblast growth factor-21 levels are associated with carotid atherosclerosis independent of established cardiovascular risk factors. Arterioscler Thromb Vasc Biol (2013) 33:2454-9. doi:10.1161/ATVBAHA.113.301599

29. Mraz M, Bartlova M, Lacinova Z, Michalsky D, Kasalicky M, Haluzikova D, et al. Serum concentrations and tissue expression of a novel endocrine regulator fibroblast growth factor-21 in patients with type 2 diabetes and obesity. Clin Endocrinol (2009) 71:369-75. doi:10.1111/j.1365-2265.2008.03502.x

30. Davis RL, Liang C, Edema-Hildebrand F, Riley C, Needham M, Sue CM. Fibroblast growth factor 21 is a sensitive biomarker of mitochondrial disease. Neurology (2013) 81:1819-26. doi:10.1212/01.wnl.0000436068.43384.ef

31. Durovcová V, Marek J, Hána V, Matoulek M, Zikán V, Haluzíková D, et al. Plasma concentrations of fibroblast growth factors 21 and 19 in patients with Cushing's syndrome. Physiol Res (2010) 59:415-22.

32. Stepan H, Kley K, Hindricks J, Kralisch S, Jank A, Schaarschmidt W. Serum levels of the adipokine fibroblast growth factor-21 are increased in preeclampsia. Cytokine (2013) 62:322-6. doi:10.1016/j.cyto.2013.02.019

33. Dostálová I, Kaválková P, Haluzíková D, Lacinová Z, Mráz M, Papezová $\mathrm{H}$, et al. Plasma concentrations of fibroblast growth factors 19 and 21 in patients with anorexia nervosa. J Clin Endocrinol Metab (2008) 3:3627-32. doi:10.1210/jc.2008-0746

Conflict of Interest Statement: The author declares that the research was conducted in the absence of any commercial or financial relationships that could be construed as a potential conflict of interest.

Received: 14 May 2014; accepted: 21 June 2014; published online: 07 July 2014. Citation: Itoh N (2014) FGF21 as a hepatokine, adipokine, and myokine in metabolism and diseases. Front. Endocrinol. 5:107. doi: 10.3389/fendo.2014.00107

This article was submitted to Diabetes, a section of the journal Frontiers in Endocrinology.

Copyright (c) 2014 Itoh. This is an open-access article distributed under the terms of the Creative Commons Attribution License (CC BY). The use, distribution or reproduction in other forums is permitted, provided the original author(s) or licensor are credited and that the original publication in this journal is cited, in accordance with accepted academic practice. No use, distribution or reproduction is permitted which does not comply with these terms. 